International Journal of Pure and Applied Mathematics

Volume 93 No. 3 2014, 463-489

ISSN: 1311-8080 (printed version); ISSN: 1314-3395 (on-line version)

url: http://www.ijpam.eu

doi: http://dx.doi.org/10.12732/ijpam.v93i3.14

ijpam.eu

\title{
GRADIENT ESTIMATES FOR HEAT-TYPE EQUATIONS ON MANIFOLDS EVOLVING BY THE RICCI FLOW
}

\author{
Abimbola Abolarinwa \\ School of Mathematical and Physical Sciences \\ University of Sussex \\ Brighton, BN1 9QH, UK
}

\begin{abstract}
In this paper, certain localized and global gradient estimates for all positive solutions to the geometric heat equation coupled to the Ricci flow either forward or backward in time are proved. As a by product, we obtain various Li-Yau type differential Harnack estimates. We also discuss the case when the diffusion operator is perturbed with the curvature operator (precisely, when the Laplacian is replaced with " $\Delta-R(x, t)$ ", $R$ being the scalar operator). This is well generalised to the case of an adjoint heat equation under the Ricci flow.
\end{abstract}

AMS Subject Classification: $35 \mathrm{~K} 05$, 53C25, 53C44

Key Words: Ricci flow, conjugate heat equation, Harnack inequalities, gradient estimates, Laplace-Beltrami operator, Laplacian comparison theorem

\section{Introduction}

Let $M$ be an $n$-dimensional compact (or noncompact without boundary) manifold on which a one parameter family of Riemannian metrics $g(t), t \in[0, T)$ is defined. We say $(M, g(t))$ is a solution to the Ricci flow if it is evolving by the following nonlinear weakly parabolic partial differential equation

Received: March 17, 2014

(c) 2014 Academic Publications, Ltd. url: www.acadpubl.eu 


$$
\frac{\partial}{\partial t} g(x, t)=-2 R c(x, t), \quad(x, t) \in M \times[0, T]
$$

with $g(x, 0)=g(0)$, where $R c$ is the Ricci curvature. By the positive solution to the heat equation on the manifold, we mean a smooth function at least $C^{2}$ in $x$ and $C^{1}$ in $t, u \in C^{2,1}(M \times[0, T])$ which satisfies the following equation

$$
\left(\Delta-\frac{\partial}{\partial t}\right) u(x, t)=0, \quad(x, t) \in M \times[0, T],
$$

where the symbol $\Delta$ is the Laplace-Beltrami operator acting on functions in space with respect to metric $g(t)$ in time. We couple the Ricci flow to the equation of the form (1.2) (either forward, backward, or perturbed with curvature operator), and obtain several gradient estimate on the logarithm of the positive solutions. We start with localized version for gradient estimate when Laplacian operator in (1.2) is replaced with $\Delta-R(x, t)$, where $R$ is the scalar curvature of the Ricci flow $g(t)$. We show how the local results imply the the global ones. As an application of our gradient estimates, we derive several Harnack estimates.

In recent years, there have been increasing efforts towards the study of the Ricci flow coupled to heat-type equation. This actually arose from R. Hamilton's work [15], where he conceived the idea of investigating Ricci flow coupled to harmonic maps heat flow. He combined this with his previous results $[13,14]$ to study the formation of singularities in the Ricci flow. In [14], he proved a Harnack estimate on Riemannian manifolds with nonnegative positive curvature operator. Hamilton also proved Harnack estimates for surfaces [12] whose positive scalar curvature under the Ricci flow satisfies the heat equation with soliton potential. B. Chow [7] completed the proof of Harnack estimate for surfaces of positive scalar curvature in general. Thus, Harnack estimates for the Ricci flow on surfaces give a control on curvature growth, while in higher dimension, one uses the Harnack estimates to classify the ancient solutions of nonnegative curvature operators. The importance of gradient estimates as well as those of Harnack inequalities can not be overemphasised in the fields of Differential geometry and Analysis among their numerous applications. Differential Harnack inequalities are used to study the behaviours of solutions to the heat equation in space-time. Li and Yau's paper [17] can be said to mark the beginning of rigorous applications of these concepts, they derived gradient estimates for positive solutions to the heat operator defined on closed manifold with bounded Ricci curvature, from which they obtained Harnack inequalities. These inequalities were in turn used to establish various lower and upper bounds on the heat kernel. They also studied manifold satisfying Dirichlet and Neumann conditions. G. Perelman [19] recorded a breakthrough in the fields of 
Topology and Geometry by the application of differential inequality, where he obtained gradient estimate for the fundamental solution to the conjugate heat equation on compact manifold evolving by the Ricci flow. Perelman's results are one of important ingredients in the proof of Poincaré conjecture. C. Guenther [11] also proved gradient estimates for positive solutions to the heat equation under the Ricci flow by adapting the methodology of Bakry and Qian [1] to time dependent metric case. As an application of her results, she got a Harnack-type inequality and obtain a lower bound for fundamental solutions. She also studied existence and basic properties of these solutions. S. Kuang and Q. Zhang [16] established a gradient estimate that holds for all positive solutions of the conjugate heat equation defined on a closed manifold whose metric is evolving by the Ricci flow. X. Cao [4] also used Perelman's approach to establish a differential inequality for all positive solutions to the conjugate heat equation also under the Ricci flow with nonnegative condition on the scalar curvature. Immediate consequences of their result in [16] and [5] are Harnack-type inequalities. Qi. Zhang [21] derived local gradient estimates for positive solutions to the heat equation coupled to the backward in time Ricci flow with assumption of lower bound on the Ricci curvature, his gradient estimate is used to prove a Gaussian bound for the conjugate heat equation. In [2], Băileşteanu, Cao, and Pulemotov applied Zhang's method to prove both space-only and space-time gradient estimates for heat equation coupled to forward in time Ricci flow. They also study manifolds with nonempty convex boundary evolving under the Ricci flow (see also [18] for a related result. Ecker, Knopf, Ni and Topping [10] have a local result on gradient estimate in relation to ean value theorem and monotonicity for heat kernel.

\subsection{Preliminaries and Notation}

We remark that our manifold is endowed with Riemannian metric $d s^{2}=g=$ $g_{i j} d x^{i} d x^{j}$, where $\left\{x^{i}\right\}, 1 \leq i \leq n$ is a local coordinate system and $n$ is the dimension of the manifold. The operator $\Delta$ is the Laplace-Beltrami operator on $(M, g)$ which is defined by

$$
\Delta_{g}=\frac{1}{\sqrt{|g|}} \partial_{i}\left(\sqrt{|g|} g^{i j} \partial_{j}\right)
$$

and $\nabla=g^{i j} \partial_{i}$ is the gradient operator, where $|g|=$ determinant of $g$ and $g^{i j}=\left(g_{i j}\right)^{-1}$, inverse metric. A natural function that will be defined on $M$ is the distance function from a given point, namely, let $p \in M$ and define $d(x, p)$ for all $x \in M$ where $\operatorname{dist}(\cdot, \cdot)$ is the geodesic distance. Note that $d(x, p)$ is only 
Lipschitz continuous, i.e., everywhere continuous except on the cut locus of $p$ and on the point where $x$ and $p$ coincide. It is then easy to see that

$$
|\nabla d|=g^{i j} \partial_{i} d \partial_{j} d=1 \quad \text { on } \quad M \backslash\{\{p\} \cup \operatorname{cut}(p)\} .
$$

Let $d(x, y, t)$ be the geodesic distance between $x$ and $y$ with respect to the metric $g(t)$, we define a smooth cut-off function $\varphi(x, t)$ with support in the geodesic cube

$$
\mathcal{Q}_{2 \rho, T}:=\{(x, t) \in M \times(0, T]: d(x, y, t) \leq 2 \rho\},
$$

for any $C^{2}$-function $\psi(s)$ on $[0,+\infty)$ with

$$
\psi(s)= \begin{cases}1, & s \in[0,1], \\ 0, & s \in[2,+\infty)\end{cases}
$$

and

$$
\psi^{\prime}(s) \leq 0, \quad \psi^{\prime \prime}(s) \geq-C_{1} \quad \text { and } \quad \frac{\left|\psi^{\prime}\right|^{2}}{\psi} \leq C_{2},
$$

where $C_{1}, C_{2}$ are absolute constants, such that

$$
\varphi(x, t)=\psi\left(\frac{d(x, p, t)}{\rho}\right) \quad \text { and }\left.\quad \varphi\right|_{\mathcal{Q}_{2 \rho, T}}=1 .
$$

We will apply maximum principle and invoke Calabi's trick to assume everywhere smoothness of $\varphi(x)$ since $\psi(s)$ is in general Lipschitz. We need Laplacian comparison theorem to do some calculation on $\varphi(x, t)$. Here is the statement of the theorem; Let $M$ be a complete $n$-dimensional Riemannian manifold whose Ricci curvature is bounded from below by $R c \geq(n-1) k$ for some constant $k \in \mathbb{R}$. Then the Laplacian of the distance function satisfies

$$
\Delta d(x, p) \leq\left\{\begin{array}{lc}
(n-1) \sqrt{k} \cot (\sqrt{k} \rho), & k>0, \\
(n-1) \rho^{-1}, & k=0, \\
(n-1) \sqrt{|k|} \operatorname{coth}(\sqrt{|k|} \rho), & k<0 .
\end{array}\right.
$$

Throughout, we will impose boundedness condition on the Ricci curvature of the metric and note that when the metric evolves by the Ricci flow, boundedness and sign assumptions are preserved as long as the flow exists, so also the metrics 
are uniformly equivalent, precisely, if $-K_{1} g \leq R c \leq K_{2} g$, where $g(t), t \in[0, T]$ is a Ricci flow, then

$$
e^{-k_{1} T} g(0) \leq g(t) \leq e^{k_{2} T} g(0) .
$$

See [8] and [9] for details on the theory of the Ricci flow. Most of our calculations are done in local coordinates where $\left\{x^{i}\right\}$ is fixed in a neighbourhood of every point $x \in M$. We switch between the $R c$ and $R_{i j}$ for the Ricci curvature notation, and any time $R_{i j}$ is used we assumed to be on local coordinates at $x$. We also define some classical identities on function $f \in C^{\infty}$ at point $x$ in a local normal coordinate system, namely,

Bochner-Weitzenböck identity

$$
\Delta|\nabla f|^{2}=2 \sum_{i j}\left|f_{i j}\right|^{2}+2 \sum_{i j} f_{j} f_{j j i}+2 \sum_{i j} R_{i j} f_{i} f_{i}
$$

and Ricci identity

$$
\sum_{i j} f_{j} f_{j i i}-\sum_{i j} f_{j} f_{j j i}=R_{i j} f_{i} f_{j}
$$

We make use of the above identities and we try as much as possible to be explicit at any point where they are used. The rest of this paper is planned as follows; In section two we discuss gradient estimate on the operator of the form $\left(\partial_{t}-\Delta+R\right)$ under both backward and forward in time Ricci flow, where we only require boundedness of scalar curvature. In section three, we discuss local space-time gradient estimates for heat equation on manifold evolving by the Ricci flow, while as an application of these results we obtain various Li-Yau type Harnack inequality in section four.

\section{The Conjugate Heat Equation under Ricci Flow}

In this section, we discuss the localized version of gradient estimate on the heat equation perturbed with curvature operator under both forward and backward Ricci flow. The estimate under backward action of Ricci flow is related to the local monotonicity for heat kernel and mean value theorem of Ecker, Knopf, Ni and Topping in [10, Section 3]. Here, they worked in general geometric flow, we follow their approach. We also remark that our result can be easily generalized to Perelman's conjugate heat equation and entropy monotonicity [19] and we can use it to prove a global Gaussian estimate as done in [21, Section 5]. 
We consider the conjugate heat equation coupled to the backward and forward Ricci flow respectively as follows

$$
\left\{\begin{array}{l}
\left(\partial_{t}-\Delta+R\right) u(x, t)=0 \\
\frac{\partial}{\partial t} g(x, t)=2 R c(x, t)
\end{array}\right.
$$

and

$$
\left\{\begin{array}{l}
\left(\partial_{t}-\Delta+R\right) u(x, t)=0 \\
\frac{\partial}{\partial t} g(x, t)=-2 \operatorname{Ric}(x, t)
\end{array}\right.
$$

Suppose $u=u(x, t)$ solves the conjugate heat equation and satisfies $0<u \leq A$ in the geodesic cube $\mathcal{Q}_{2 \rho} \subset M$ as defined by

$$
\mathcal{Q}_{2 \rho, T}:=\{(x, t) \in M \times(0, T]: d(x, y, t) \leq 2 \rho\}
$$

then we have

Theorem 2.1. Let $(M, g(t)), t \in[0, T]$ be a complete solution to the backward Ricci flow (2.1b) with $R \geq-k_{1}, R c \geq-k_{2}$ and $|\nabla R| \leq k_{3}$, for some constants $k_{1}, k_{2}, k_{3} \geq 0$. Let $u=u(x, t)$ be any positive solution to the heat equation (2.1a)defined in $\mathcal{Q}_{2 \rho, T} \subset(M \times[0, T])$, satisfying $0<u \leq A$. There exist absolute constants $C_{1}, C_{2}$ depending on $n$ such that

$$
\frac{|\nabla u|^{2}}{u^{2}} \leq\left(1+\log \left(\frac{A}{u}\right)\right)^{2}\left(\frac{1}{t}+C_{2} k_{1}+4 k_{2}+2 k_{3}+\frac{1}{\rho^{2}}\left(\rho C_{1} \sqrt{k_{2}}+C_{2}\right)\right) .
$$

The boundedness assumption may be weakened in the case of the forward Ricci flow (2.2) as we can state our result as follows;

Theorem 2.2. Let $(M, g(t)), t \in[0, T]$ be a complete solution to the forward Ricci flow (2.2b) with $R \geq-k_{1}$ and $|\nabla R| \leq k_{3}$, for some constants $k_{1}, k_{2}, k_{3} \geq 0$. Let $u=u(x, t)$ be any positive solution to the heat equation (2.2a)defined in $\mathcal{Q}_{2 \rho, T} \subset(M \times[0, T])$, satisfying $0<u \leq A$. There exist absolute constants $C_{1}, C_{2}$ depending on $n$ such that

$$
\frac{|\nabla u|^{2}}{u^{2}} \leq\left(1+\log \left(\frac{A}{u}\right)\right)^{2}\left(\frac{1}{t}+C_{2} k_{1}+2 k_{3}+\frac{C_{2}}{\rho^{2}}\right) .
$$

Proof. (of Theorem 2.1)

Let $f=\log u$ and $w=|\nabla \ln (1-f)|^{2}=\frac{|\nabla f|^{2}}{(1-f)^{2}}$. By standard calculation, we have $\partial_{t} f=\Delta f+|\nabla f|^{2}-R$ and

$$
\partial_{t}\left(|\nabla f|^{2}\right)=\left(\partial_{t} g^{i j}\right) \partial_{i} f \partial_{j} f+2\left\langle\nabla f, \nabla \partial_{t} f\right\rangle
$$




$$
\begin{aligned}
& =-2 R_{i j} f_{i} f_{j}+2\left\langle\nabla f, \nabla\left(\Delta f+|\nabla f|^{2}-R\right)\right\rangle \\
& =-2 R_{i j} \nabla_{i} f \nabla_{j} f+2\langle\nabla f, \nabla \Delta f\rangle+2\left\langle\nabla f, \nabla|\nabla f|^{2}\right\rangle-2\langle\nabla f, \nabla R\rangle .
\end{aligned}
$$

By Bochner-Weitzenböck identity (1.6), we have

$$
\Delta\left(|\nabla f|^{2}\right)=2|\nabla \nabla f|^{2}+2\langle\nabla f, \nabla \Delta f\rangle+2 R_{i j} f_{i} f_{j}
$$

Then

$$
\left(\partial_{t}-\Delta\right)|\nabla f|^{2}=-2 f_{i j}^{2}+2\left\langle\nabla f, \nabla|\nabla f|^{2}\right\rangle-2\langle\nabla f, \nabla R\rangle-4 R_{i j} \nabla_{i} f \nabla_{j} f
$$

Next we compute

$$
\begin{gathered}
w_{t}=\frac{\partial}{\partial t}\left(\frac{|\nabla f|^{2}}{(1-f)^{2}}\right)=\frac{\partial_{t}|\nabla f|^{2}}{(1-f)^{2}}+\frac{2|\nabla f|^{2} \partial_{t} f}{(1-f)^{3}} \\
=\frac{-2 R_{i j} \nabla_{i} f \nabla_{j} f+2\langle\nabla f, \nabla \Delta f\rangle+2\left\langle\nabla f, \nabla|\nabla f|^{2}\right\rangle-2\langle\nabla f, \nabla R\rangle}{(1-f)^{2}} \\
+\frac{2|\nabla f|^{2}\left(\Delta f+|\nabla f|^{2}-R\right)}{(1-f)^{3}} \\
=-\frac{2 R_{i j} \nabla_{i} f \nabla_{j} f}{(1-f)^{2}}+\frac{2 f_{j} f_{j j i}}{(1-f)^{2}}+\frac{2\left\langle\nabla f, \nabla|\nabla f|^{2}\right\rangle}{(1-f)^{2}}-\frac{2\langle\nabla f, \nabla R\rangle}{(1-f)^{2}} \\
+\frac{2|\nabla f|^{2} \Delta f}{(1-f) 3}+\frac{2|\nabla f|^{4}}{(1-f)^{3}}-\frac{2 R|\nabla f|^{2}}{(1-f)^{3}} .
\end{gathered}
$$

Compute $\Delta w$

$$
\begin{gathered}
\nabla w=\nabla\left(\frac{|\nabla f|^{2}}{(1-f)^{2}}\right)=\frac{2\langle\nabla f, \nabla \nabla f\rangle}{(1-f)^{2}}+\frac{2|\nabla f|^{2} \nabla f}{(1-f)^{3}} \\
=\frac{2 f_{i} f_{i j}}{(1-f)^{2}}+\frac{2 f_{i}^{2} f_{j}}{(1-f)^{3}} . \\
\Delta w=\left(\frac{2 f_{i} f_{i j}}{(1-f)^{2}}\right)_{j}+\left(\frac{2 f_{i}^{2} f_{j}}{(1-f)^{3}}\right)_{j} \\
=\frac{2\left(f_{i j} f_{i j}+f_{i} f_{i j j}\right)}{(1-f)^{2}}+\frac{4(1-f) f_{i} f_{i j} f_{j}}{(1-f)^{4}}+\frac{4 f_{i} f_{i j} f_{j}+2 f_{i}^{2} f_{j j}}{(1-f)^{3}}+\frac{6 f_{i}^{2} f_{j}^{2}(1-f)}{(1-f)^{6}}
\end{gathered}
$$




$$
=\frac{2 f_{i j}^{2}}{(1-f)^{2}}+\frac{2 f_{i} f_{i j j}}{(1-f)^{2}}+\frac{4 f_{i} f_{i j} f_{j}}{(1-f)^{3}}+\frac{4 f_{i} f_{i j} f_{j}}{(1-f)^{3}}+\frac{2 f_{i}^{2} f_{j j}}{(1-f)^{3}}+\frac{6 f_{i}^{2} f_{j}^{2}}{(1-f)^{4}} .
$$

Hence

$$
\begin{aligned}
\left(\partial_{t}-\Delta\right) w & =-\frac{2 R_{i j} f_{i} f_{j}}{(1-f)^{2}}+\frac{2 f_{j} f_{j j i}}{(1-f)^{2}}+\frac{2\left\langle\nabla f, \nabla|\nabla f|^{2}\right\rangle}{(1-f)^{2}}-\frac{2\langle\nabla f, \nabla R\rangle}{(1-f)^{2}}+\frac{2|\nabla f|^{4}}{(1-f)^{3}} \\
& -\frac{2 R|\nabla f|^{2}}{(1-f)^{3}}-\frac{2 f_{i j}^{2}}{(1-f)^{2}}-\frac{2 f_{i} f_{i j j}}{(1-f)^{2}}-\frac{4 f_{i} f_{i j} f_{j}}{(1-f)^{3}}-\frac{4 f_{i} f_{i j} f_{j}}{(1-f)^{2}}-\frac{6 f_{i}^{2} f_{j}^{2}}{(1-f)^{4}} .
\end{aligned}
$$

Rearranging

$$
\begin{aligned}
& \left(\partial_{t}-\Delta\right) w \\
& =\underbrace{-\frac{2}{(1-f)^{2}}\left(f_{i j}^{2}+\frac{2 f_{i} f_{i j} f_{j}}{1-f}+\frac{f_{i}^{2} f_{j}^{2}}{(1-f)^{2}}\right)}-\underbrace{\frac{2 R_{i j} f_{i} f_{j}}{(1-f)^{2}}+\frac{2 f_{j} f_{j j i}}{(1-f)^{2}}-\frac{2 f_{i} f_{i j j}}{(1-f)^{2}}} \\
& -\underbrace{\frac{4 f_{i} f_{i j} f_{j}}{(1-f)^{3}}-\frac{4 f_{i}^{2} f_{j}^{2}}{(1-f)^{4}}+\frac{2\left\langle\nabla f, \nabla|\nabla f|^{2}\right\rangle}{(1-f)^{2}}}-\underbrace{\frac{2\langle\nabla f, \nabla R\rangle}{(1-f)^{2}}-\frac{2 R|\nabla f|^{2}}{(1-f)^{3}}+\frac{2|\nabla f|^{4}}{(1-f)^{3}}}
\end{aligned}
$$

Note that we can simplify some terms in the last equation further. The first term becomes

$$
-\frac{2}{(1-f)^{2}}\left(f_{i j}^{2}+\frac{2 f_{i} f_{i j} f_{j}}{1-f}+\frac{f_{i}^{2} f_{j}^{2}}{(1-f)^{2}}\right)=-\frac{2}{(1-f)^{2}}\left(f_{i j}+\frac{f_{i} f_{j}}{1-f}\right)^{2} .
$$

Using the Ricci identity on the next three terms we have

$$
-\frac{2 R_{i j} f_{i} f_{j}}{(1-f)^{2}}+\frac{2 f_{j} f_{j j i}}{(1-f)^{2}}-\frac{2 f_{i} f_{i j j}}{(1-f)^{2}}=-\frac{4 R_{i j} f_{i} f_{j}}{(1-f)^{2}},
$$

since the Ricci identity (1.7) implies

$$
f_{j} f_{j j i}-f_{i} f_{i j j}=f_{j}\left(f_{j j i}-f_{i j j}\right)=-R_{i j} f_{i} f_{j} .
$$

Also

$$
-\frac{4 f_{i} f_{i} j f_{j}}{(1-f)^{3}}-\frac{4 f_{i}^{2} f_{j}^{2}}{(1-f)^{4}}=\frac{-2}{1-f} f_{j}\left(\frac{2 f_{i} f_{i j}}{(1-f)^{2}}+\frac{2 f_{i}^{2} f_{j}}{(1-f)^{3}}\right)=\frac{-2}{1-f}\langle\nabla f, \nabla w\rangle,
$$

similarly,

$$
\frac{2\left\langle\nabla f, \nabla|\nabla f|^{2}\right\rangle}{(1-f)^{2}}=2\langle\nabla f, \nabla w\rangle
$$


then the second to the last three terms gives

$$
\begin{aligned}
-\frac{4 f_{i} f_{i j} f_{j}}{(1-f)^{3}}-\frac{4 f_{i}^{2} f_{j}^{2}}{(1-f)^{4}}+\frac{2\left\langle\nabla f, \nabla|\nabla f|^{2}\right\rangle}{(1-f)^{2}} & =\frac{-2}{1-f}\langle\nabla f, \nabla w\rangle+2\langle\nabla f, \nabla w\rangle \\
& =\frac{-2 f}{1-f}\langle\nabla f, \nabla w\rangle .
\end{aligned}
$$

Putting all these together, we get

$$
\begin{aligned}
\left(\partial_{t}-\Delta\right) w & =-\frac{2}{(1-f)^{2}}\left(f_{i j}+\frac{f_{i} f_{j}}{1-f}\right)^{2}-\frac{2 f}{1-f}\langle\nabla f, \nabla w\rangle-\frac{4 R_{i j} f_{i} f_{j}}{(1-f)^{2}} \\
& -\frac{2\langle\nabla f, \nabla R\rangle}{(1-f)^{2}}-\frac{2 R|\nabla f|^{2}}{(1-f)^{3}}-\frac{2|\nabla f|^{4}}{(1-f)^{3}},
\end{aligned}
$$

then using the curvature conditions we are left with the following inequality

$$
\left(\partial_{t}-\Delta\right) w \leq-\frac{2 f}{1-f}\langle\nabla f, \nabla w\rangle+\frac{2}{1-f} k_{1} w+4 k_{2} w+2 k_{3} w^{\frac{1}{2}}-2(1-f) w^{2} .
$$

We now apply a cut-off function in order to derive the desired estimate. Now, let $\psi(s)$ be a smooth cut function defined on $[0, \infty)$ such that $0 \leq \psi(s) \leq 1$, with

$$
\psi^{\prime}(s) \leq 0, \quad \psi^{\prime \prime}(s) \geq-C_{1} \quad \text { and } \quad \frac{\left|\psi^{\prime}\right|^{2}}{\psi} \leq C_{2}
$$

for some constants $C_{1}, C_{2}>0$ depending on the dimension of the manifold only. Define a distance function $d(p, x)$ between points $p$ and $x$ such that

$$
\varphi(x, t)=\varphi(d(p, x, t))=\psi\left(\frac{d_{g(t)}(p, x)}{\rho}\right)
$$

for smooth function $\varphi: M \times[0, T] \rightarrow \mathbb{R}$.

It is easily seen that $\varphi(x, t)$ has its support in the closure of $\mathcal{Q}_{2 \rho, T}$. We note that $\varphi(x, t)$ is smooth at $(y, s) \in M \times[0, T]$ whenever point $y$ does not either coincide with $p$ or fall in the cut locus of $p$, with respect to the metric $g(y, s)$. In what follows, we consider the function $\varphi w$ supported in $\mathcal{Q}_{2 \rho, T} \times[0, \infty)$, though, this is in general Lipchitz continuous. For the purpose of the application of the maximum principle, we may therefore, without loss of generality assume that $\varphi(x, t)$ with support in $\mathcal{Q}_{2 \rho, T}$ is $C^{2}$ at the maxima. The assumption is made possible by a standard argument called Calabi's trick (due to Calabi 1958 [3]). This approach is used in [6], see also [10, 20, 21]. Therefore we can calculate

$$
\frac{|\nabla \varphi|^{2}}{\varphi}=\frac{\left|\psi^{\prime}\right|^{2} \cdot|\nabla d|^{2}}{\rho^{2} \varphi} \leq \frac{C_{2}}{\rho^{2}}, \quad \frac{\partial \varphi}{\partial t} \leq C_{2} k_{1}
$$


and by the Laplacian comparison theorem we have

$$
\begin{aligned}
\Delta \varphi & =\frac{\psi^{\prime} \Delta d}{\rho}+\frac{\psi^{\prime \prime}|\nabla d|^{2}}{\rho} \\
& \geq-\frac{C_{1}}{\rho}(n-1) \sqrt{k_{2}} \operatorname{coth}\left(\sqrt{k_{2}} \rho\right)-\frac{C_{2}}{\rho^{2}} \\
\Longrightarrow-\Delta \varphi & \leq \frac{1}{\rho^{2}}\left(C_{1} \sqrt{k_{2}} \rho \operatorname{coth}\left(\sqrt{k_{2}} \rho\right)+C_{2}\right) .
\end{aligned}
$$

Let $\left(x_{0}, t_{0}\right)$ be a point in $\mathcal{Q}_{2 \rho, T}$ at which $F=\varphi w$ attains its maximum value. At this point we have to assume that $F$ is positive, since, if $F=0$, implies $\varphi w\left(x_{0}, t_{0}\right)=0 \Longrightarrow \varphi w(x, t)=0 \Longrightarrow w(x, t)=0$ for all $x \in M$ such that the distance $d\left(x, x_{0}, t\right)<2 \rho$, this yields $\nabla u(x, t)=0$ and the theorem will follow trivially at $(x, t)$. The approach here is to estimate $\left(\partial_{t}-\Delta\right)(t F)$ and do some analysis on the result at the maximum point. The argument is as follows

$$
\begin{aligned}
\left(\partial_{t}-\Delta\right)(t F) & =F+t\left(\partial_{t}-\Delta\right)(\varphi w) \\
& =F-2 t\langle\nabla \varphi, \nabla w\rangle+t \varphi\left(\partial_{t}-\Delta\right) w+t w\left(\partial_{t}-\Delta\right) \varphi
\end{aligned}
$$

Note that at the maximum point $\left(x_{0}, t_{0}\right)$, we have by derivative test that

$$
\nabla F\left(x_{0}, t_{0}\right)=0, \quad \frac{\partial}{\partial t} F\left(x_{0}, t_{0}\right) \geq 0 \quad \text { and } \quad \Delta F\left(x_{0}, t_{0}\right) \leq 0 .
$$

Taking $t F$ on $M \times[0, T]$, we have $\left(\partial_{t}-\Delta\right)(t F) \geq 0$ whenever $(t F)$ achieves its maximum. Similarly, by this argument we have

$$
\nabla(\varphi w)\left(x_{0}, t_{0}\right)-w \nabla \varphi\left(x_{0}, t_{0}\right)=\varphi \nabla w\left(x_{0}, t_{0}\right)
$$

which means $\varphi \nabla w$ can always be replaced by $-w \nabla \varphi$. By $(2.9)$, ( 2.14) and ( 2.16)

$$
\begin{aligned}
0 \leq F & -2 t\langle\nabla \varphi, \nabla w\rangle \\
& +t \varphi\left\{-\frac{2 f}{1-f}\langle\nabla f, \nabla w\rangle+\frac{2}{1-f} k_{1} w+4 k_{2} w+2 k_{3} w^{\frac{1}{2}}+2(1-f) w^{2}\right\} \\
& +t w\left(\partial_{t}-\Delta\right) \varphi
\end{aligned}
$$

Taking $0 \leq \varphi \leq 1$ and noticing that $\frac{1}{1-f} \leq 1$, then the last inequality becomes

$$
0 \leq F-2 t \frac{\nabla \varphi}{\varphi} \cdot \nabla(\varphi w)-2 t \frac{|\nabla \varphi|^{2}}{\varphi} w
$$




$$
\begin{aligned}
& +t\left\{2 k_{1} F+4 k_{2} F+2 k_{3} F^{\frac{1}{2}}+2(1-f) F^{2}\right\}+2 t\left(-\frac{f}{1-f}\right)|\nabla f||\nabla \varphi| w \\
& +t w\left(\partial_{t}-\Delta\right) \varphi .
\end{aligned}
$$

Using the following relation as noticed in [10];

$$
2 k_{3} F^{\frac{1}{2}} \leq K_{3} F+k_{3}
$$

and by the Young's inequlity

$$
\begin{aligned}
2 t\left(-\frac{f}{1-f}\right)|\nabla f||\nabla \varphi| w & \leq t w\left(\frac{|\nabla f|^{2}}{1-f} \varphi+\frac{|\nabla \varphi|^{2}}{\varphi} \frac{f^{2}}{1-f}\right) \\
& =t \varphi(1-f) w^{2}+t w \frac{|\nabla \varphi|^{2}}{\varphi} \frac{f^{2}}{1-f} \\
& =t(1-f) F^{2}+t F \frac{C_{2}}{\rho^{2}} \frac{f^{2}}{1-f} .
\end{aligned}
$$

Notice also that by bounds given in (2.10) and (2.11) we have

$$
\begin{aligned}
t w\left(\partial_{t}-\Delta\right) \varphi & \leq t w\left(C_{2} k_{1}+\frac{1}{\rho^{2}}\left(C_{1} \sqrt{k_{2}} \operatorname{coth}\left(\sqrt{k_{2}} \rho\right)+C_{2}\right)\right) \\
& \leq t w\left(C_{2} k_{1}+\frac{1}{\rho^{2}}\left(\rho C_{1} \sqrt{k_{2}}+C_{2}\right)\right) .
\end{aligned}
$$

Putting these together and dividing through by $(1-f)$, while noticing that $\frac{1}{1-f} \leq 1$ and $\frac{-f}{1-f} \leq 1$, we have

$$
\begin{aligned}
0 \leq F & -2 t \frac{C_{2}}{\rho^{2}} F+t\left(2 k_{1}+4 k_{2}+k_{3}\right) F+t k_{3}+t F \frac{C_{2}}{\rho^{2}}-t F^{2} \\
& +t w\left(C_{2} k_{1}+\frac{1}{\rho^{2}}\left(\rho C_{1} \sqrt{k_{2}}+C_{2}\right)\right) .
\end{aligned}
$$

Therefore we have

$$
\begin{gathered}
t F^{2} \leq F+t\left(2 k_{1}+4 k_{2}+k_{3}\right) F \\
+t\left(C_{2} k_{1}+\frac{1}{\rho^{2}}\left(\rho C_{1} \sqrt{k_{2}}+C_{2}\right)\right) F-t \frac{C_{2}}{\rho^{2}} F+t k_{3} \\
F^{2} \leq F\left\{\frac{1}{t}+C_{2} K_{1}+4 k_{2}+k_{3}+\frac{1}{\rho^{2}}\left(\rho C_{1} \sqrt{k_{2}}+C_{2}\right)+k_{3}\right\}+k_{3} .
\end{gathered}
$$

From here we can conclude that

$$
F \leq \frac{1}{t}+C_{2} K_{1}+4 k_{2}+k_{3}+\sqrt{k_{3}}+\frac{1}{\rho^{2}}\left(\rho C_{1} \sqrt{k_{2}}+C_{2}\right)
$$


at $\left(x_{0}, t_{0}\right)$. Therefore

$$
|\nabla f|^{2} \leq(1-f)^{2}\left(\frac{1}{t}+C_{2} K_{1}+4 k_{2}+2 k_{3}+\frac{1}{\rho^{2}}\left(\rho C_{1} \sqrt{k_{2}}+C_{2}\right)\right) .
$$

By standard scaling argument we can assume $A \equiv 1$, hence the proof is completed.

\section{Proof. (of Theorem 2.2)}

The proof of Theorem 2.2 is similar to that of Theorem 2.1, the disparity between the estimates (2.4) and (2.5) arises in some calculation which we briefly point out here.

Similarly, set $f=\log u$ and $w=\frac{|\nabla f|^{2}}{(1-f)^{2}}$. Notice that $g(t)$ evolves by the Ricci flow (2.2), where the inverse metric evolves as $\partial_{t}\left(g^{i j}\right)=2 R_{i j}$ and then

$$
\partial_{t}\left(|\nabla f|^{2}\right)=2 R_{i j} \nabla_{i} f \nabla_{j} f+2\langle\nabla f, \nabla \Delta f\rangle+2\left\langle\nabla f, \nabla|\nabla f|^{2}\right\rangle-2\langle\nabla f, \nabla R\rangle
$$

hence, the counterpart of $(2.8)$ is

$$
\begin{aligned}
& \left(\partial_{t}-\Delta\right) w \\
& =\underbrace{-\frac{2}{(1-f)^{2}}\left(f_{i j}^{2}+\frac{2 f_{i} f_{i j} f_{j}}{1-f}+\frac{f_{i}^{2} f_{j}^{2}}{(1-f)^{2}}\right)}+\underbrace{\frac{2 R_{i j} f_{i} f_{j}}{(1-f)^{2}}+\frac{2 f_{j} f_{j j i}}{(1-f)^{2}}-\frac{2 f_{i j}^{2}}{(1-f)^{2}}} \\
& -\underbrace{\frac{4 f_{i} f_{i j} f_{j}}{(1-f)^{3}}-\frac{4 f_{i}^{2} f_{j}^{2}}{(1-f)^{4}}+\frac{2\left\langle\nabla f, \nabla|\nabla f|^{2}\right\rangle}{(1-f)^{2}}}-\underbrace{\frac{2\langle\nabla f, \nabla R\rangle}{(1-f)^{2}}-\frac{2 R|\nabla f|^{2}}{(1-f)^{3}}+\frac{2|\nabla f|^{4}}{(1-f)^{3}}}
\end{aligned}
$$

Using the Ricci identity, the second braced terms vanish and we are left with following inequality as in (2.9)

$$
\left(\partial_{t}-\Delta\right) w \leq-\frac{2 f}{1-f}\langle\nabla f, \nabla w\rangle+\frac{2}{1-f} k_{1} w+2 k_{3} w^{\frac{1}{2}}-2(1-f) w^{2} .
$$

We then proceed as in the the rest of the proof of theorem (2.1).

This can be seen as an improvement since the curvature assumption is weakened, we only require boundedness of scalar curvature. Global estimates can be obtained from the two gradient estimates, simply by sending $\rho$ to $\infty$. For instance, when $u$ is globally defined and $g(t)$ has nonnegative curvature, the estimate simply read as

$$
\frac{|\nabla u|^{2}}{u^{2}} \leq\left(1+\frac{A}{u}\right)^{2}\left(\frac{1}{t}+2 k_{3}\right) .
$$




\section{Gradient Estimates on Forward Heat equation}

Let $\mathrm{M}$ be an $n$-dimensional complete Riemannian manifold without boundary, we discuss space-time gradient estimates for positive solutions of the heat equation along the Ricci flow

$$
\frac{\partial}{\partial t} g(x, t)=-2 R(x, t), \quad(x, t) \in M \times[0, T]
$$

coupled to

$$
\left(\Delta-\frac{\partial}{\partial t}\right) u(x, t)=0, \quad(x, t) \in M \times[0, T] .
$$

In general, our estimate is a local one as we obtain it in the interior of geodesic cube. We will show how this local estimate can lead to achieving a global one. We first proof a lemma which is very crucial to our derivation. Define the geodesic cube

$$
\mathcal{Q}_{2 \rho, T}:=\left\{(x, t) \in M \times(0, T]: d\left(x, x_{0}, t\right) \leq \rho\right\} .
$$

Lemma 3.1. Let $(M, g(t))$ be a complete solution to the Ricci flow in some time interval $[0, T]$. Suppose that $-k_{1} g \leq R c \leq k_{2} g$ for some positive constants $k_{1}$ and $k_{2}$ and for all $t \in[0, T]$. For any smooth positive solution $u \in C^{2,1}(M \times[0, T])$ to the heat equation in the geodesic cube $\mathcal{Q}_{2 \rho, T}$, it holds that

$$
\begin{aligned}
\left(\Delta-\partial_{t}\right) G \geq & -2\langle\nabla f, \nabla G\rangle-\left(|\nabla f|^{2}-\alpha \partial_{t} f\right)-2 \alpha t k_{1}|\nabla f|^{2} \\
& +\frac{2 \alpha}{n p} t\left(|\nabla f|^{2}-\partial_{t} f\right)^{2}-\frac{\alpha n q}{2} t\left(k_{1}+k_{2}\right)^{2},
\end{aligned}
$$

where $f=\log u, G=t\left(|\nabla f|^{2}-\alpha \partial_{t} f\right)$ and $\alpha \geq 1$ are given such that $\frac{1}{p}+\frac{1}{q}=\frac{1}{\alpha}$ for any real numbers $p, q>0$.

Proof. We have

$$
\begin{aligned}
\Delta G & =t\left(\Delta|\nabla f|^{2}-\alpha \Delta \partial_{t} f\right) \\
\partial_{t} G & =\left(|\nabla f|^{2}-\alpha \partial_{t} f\right)+t\left(|\nabla f|^{2}-\alpha \partial_{t} f\right)_{t} .
\end{aligned}
$$

Working in local coordinates system at any point $x \in M$ and using Einstein summation convection where repeated indices are summed up. We have by Bochner-Weitzenböck's identity (1.6)

$$
\Delta|\nabla f|^{2}=2 f_{i j}^{2}+2 f_{j} f_{j j i}+2 R_{i j} f_{i} f_{j} .
$$


By the hypothesis of the lemma that $g(x, t)$ evolves by the Ricci flow we have

$$
\begin{aligned}
\partial_{t}\left(|\nabla f|^{2}\right) & =\left(\partial_{t} g^{i j}\right) \partial_{i} f \partial_{j} f+2 g^{i j} \partial_{i} f \partial_{j} \partial_{t} f \\
& =2 R_{i j} f_{i} f_{j}+2 f_{i} f_{t, i}
\end{aligned}
$$

similarly,

$$
\begin{aligned}
\partial_{t}(\Delta f) & =\left(\partial_{t} g^{i j}\right) \partial_{i} \partial_{j} f+g^{i j} \partial_{i} \partial_{j} \partial_{t} f \\
& =2 R_{i j} f_{i j}+\Delta f_{t}, \\
\text { which } \Longrightarrow \Delta f_{t} & =(\Delta f)_{t}-2 R_{i j} f_{i j} .
\end{aligned}
$$

With the above computations we obtain the following at an arbitrary point $(x, t) \in \mathcal{Q}_{2 \rho, T}$

$$
\begin{aligned}
\Delta G & =t\left(2 f_{i j}^{2}+2 f_{i} f_{j j i}+2 R_{i j} f_{i} f_{j}+2 \alpha R_{i j} f_{i j}-\alpha(\Delta f)_{t}\right) \\
& \geq t\left[\left(2 f_{i j}^{2}+2 \alpha R_{i j} f_{i j}\right)+2 f_{j} f_{j j i}-2 k_{1}|\nabla f|^{2}-\alpha(\Delta f)_{t}\right] .
\end{aligned}
$$

On the other hand

$$
\partial_{t} G=\left(|\nabla f|^{2}-\alpha \partial_{t} f\right)+t\left(2 R_{i j} f_{i} f_{j}+2 f_{i} f_{t, i}-\alpha f_{t t}\right) .
$$

Noticing that $f=\log u$ implies the evolution

$$
\partial_{t} f=\Delta f+|\nabla f|^{2}, \quad(x, t) \in \mathcal{Q}_{2 \rho, T} .
$$

Therefore

$$
\begin{aligned}
& \left(\Delta-\partial_{t}\right) G \geq t\left[\left(2 f_{i j}^{2}+2 \alpha R_{i j} f_{i j}\right)+2 f_{j} f_{j j i}-2 k_{1}|\nabla f|^{2}-\alpha(\Delta f)_{t}\right] \\
& \quad-\left[\left(|\nabla f|^{2}-\alpha \partial_{t} f\right)+t\left(|\nabla f|^{2}-\alpha \partial_{t} f\right)_{t}\right] \\
& =t\left(2 f_{i j}^{2}+2 \alpha R_{i j} f_{i j}\right)+2 t\left\langle\nabla f, \nabla\left(\partial_{t} f-|\nabla f|^{2}\right)\right\rangle-2 t k_{1}|\nabla f|^{2} \\
& \quad-\alpha\left(\partial_{t} f-|\nabla f|^{2}\right)_{t}-\left(|\nabla f|^{2}-\alpha \partial_{t} f\right)-t\left(|\nabla f|^{2}-\alpha \partial_{t} f\right)_{t} \\
& =t\left(2 f_{i j}^{2}+2 \alpha R_{i j} f_{i j}\right)-2 t\left\langle\nabla f, \nabla\left(|\nabla f|^{2}-\alpha \partial_{t} f\right)\right\rangle-2 t\left\langle\nabla f, \nabla\left(\alpha \partial_{t} f-\partial_{t} f\right)\right\rangle \\
& \quad-2 t k_{1}|\nabla f|^{2}-\alpha t\left(\partial_{t} f-|\nabla f|^{2}\right)_{t}-\left(|\nabla f|^{2}-\alpha \partial_{t} f\right)-t\left(|\nabla f|^{2}-\alpha \partial_{t} f\right)_{t} \\
& =t\left(2 f_{i j}^{2}+2 \alpha R_{i j} f_{i j}\right)-2\langle\nabla f, \nabla G\rangle-\left(|\nabla f|^{2}-\alpha \partial_{t} f\right)-2 t k_{1}|\nabla f|^{2} \\
& \quad-2(\alpha-1) t\left\langle\nabla f, \nabla \partial_{t} f\right\rangle-\alpha t\left(\partial_{t} f-|\nabla f|^{2}\right)_{t}-t\left(|\nabla f|^{2}-\alpha \partial_{t} f\right)_{t}
\end{aligned}
$$




$$
\begin{gathered}
=t\left(2 f_{i j}^{2}+2 \alpha R_{i j} f_{i j}\right)-2\langle\nabla f, \nabla G\rangle-\left(|\nabla f|^{2}-\alpha \partial_{t} f\right)-2 t k_{1}|\nabla f|^{2} \\
\quad-2(\alpha-1) t\left\langle\nabla f, \nabla \partial_{t} f\right\rangle+(\alpha-1) t\left(|\nabla f|^{2}\right)_{t} \\
=t\left(2 f_{i j}^{2}+2 \alpha R_{i j} f_{i j}\right)-2\langle\nabla f, \nabla G\rangle-\left(|\nabla f|^{2}-\alpha \partial_{t} f\right)-2 t k_{1}|\nabla f|^{2} \\
\quad-2(\alpha-1) t\left\langle\nabla f, \nabla \partial_{t} f\right\rangle-(\alpha-1) t\left[2 R_{i j} f_{i j}+2\left\langle\nabla f, \nabla \partial_{t} f\right\rangle\right] \\
\geq t\left(2 f_{i j}^{2}+2 \alpha R_{i j} f_{i j}\right)-2\langle\nabla f, \nabla G\rangle-\left(|\nabla f|^{2}-\alpha \partial_{t} f\right)-2 t k_{1}|\nabla f|^{2} \\
\quad-2(\alpha-1) t\left\langle\nabla f, \nabla \partial_{t} f\right\rangle-2(\alpha-1) t k_{1}|\nabla f|^{2}+2(\alpha-1) t\left\langle\nabla f, \nabla \partial_{t} f\right\rangle \\
=t\left(2 f_{i j}^{2}+2 \alpha R_{i j} f_{i j}\right)-2\langle\nabla f, \nabla G\rangle-\left(|\nabla f|^{2}-\alpha \partial_{t} f\right)-2 \alpha t k_{1}|\nabla f|^{2} .
\end{gathered}
$$

Now we can choose any two real numbers $p, q>0$ such that $\frac{1}{p}+\frac{1}{q}=\frac{1}{\alpha}$, so that we can write

$$
\begin{aligned}
2 f_{i j}^{2}+2 \alpha R_{i j} f_{i j} & =\frac{2 \alpha}{p} f_{i j}^{2}+2 \alpha\left(\frac{1}{q} f_{i j}^{2}+R_{i j} f_{i j}\right) \\
& =\frac{2 \alpha}{p} f_{i j}^{2}+2 \alpha\left(\frac{1}{\sqrt{q}} f_{i j}+\frac{\sqrt{q}}{2} R_{i j}\right)^{2}-\frac{\alpha q}{2} R_{i j}^{2} \\
& \geq \frac{2 \alpha}{p} f_{i j}^{2}-\frac{\alpha q}{2} R_{i j}^{2},
\end{aligned}
$$

where we have used completing the square method to arrive at the last inequality. Also by Cauchy-Schwarz inequality

$$
(\Delta f)^{2}=\left|g^{i j} \partial_{i} \partial_{j} f\right|^{2} \leq n f_{i j}^{2}
$$

holds at an arbitrary point $(x, t) \in \mathcal{Q}_{2 \rho, T}$, therefore we have $f_{i j}^{2} \geq \frac{1}{n}(\Delta f)^{2}$. We can also write the boundedness condition on the Ricci curvature as $-\left(k_{1}+\right.$ $\left.k_{2}\right) g \leq R_{i j} \leq\left(k_{1}+k_{2}\right) g$ so that

$$
\sup _{M}\left|R_{i j}\right|^{2} \leq n\left(k_{1}+k_{2}\right)^{2}
$$

since the Ricci curvature tensor is symmetric. Therefore

$$
2 t\left(f_{i j}^{2}+\alpha R_{i j} f_{i j}\right) \geq \frac{2 \alpha}{n p} t(\Delta f)^{2}-\frac{\alpha n q}{2} t\left(k_{1}+k_{2}\right)^{2} .
$$

Hence the result. Our calculation is valid in the cube $\mathcal{Q}_{2 \rho, T}$.

In the next, we state and prove a result for local gradient estimate (spacetime) for the positive solutions to the heat equation in the geodesic cube $\mathcal{Q}_{2 \rho, T}$ of bounded Ricci curvature manifold evolving by the Ricci flow. 
Theorem 3.2. Let $(M, g(t)), t \in[0, T]$ be a complete solution of the Ricci flow ( 3.1) such that the Ricci curvature is bounded in $\mathcal{Q}_{2 \rho, T}$, i.e., $-k_{1} g(x, t) \leq$ $R c(x, t) \leq k_{2} g(x, t)$ for some positive constants $k_{1}$ and $k_{2}$, with $(x, t) \in \mathcal{Q}_{2 \rho, T} \subset$ $(M \times[0, T])$. Suppose a smooth positive function $u \in C^{2,1}(M \times[0, t])$ solves the heat equation (3.2) in the cube $\mathcal{Q}_{2 \rho, T}$. Then, for any given $\alpha>1$ with $\frac{1}{p}+\frac{1}{q}=\frac{1}{\alpha}$ and all $(x, t) \in \mathcal{Q}_{2 \rho} \times[0, T]$, the following estimate holds

$$
\left.\begin{array}{rl}
\sup _{x \in \mathcal{Q}_{2 \rho}}\left\{|\nabla f|^{2}-\alpha f_{t}\right\} \leq & \frac{\alpha n p}{4 t}+C \alpha^{2}\left(\frac{\alpha^{2} p}{\rho^{2}(\alpha-1)}+\frac{1}{t}+\left(k_{1}+k_{2}\right)\right) \\
& +\frac{\alpha^{2} n p}{2(\alpha-1)} k_{1}+\frac{\alpha n}{2}\left(k_{1}+k_{2}\right) \sqrt{p q}
\end{array}\right\},
$$

where $C$ is an arbitrary constant depending only on the dimension of the manifold.

Proof. Note that as before we let $f=\log u$ and $G=t\left(|\nabla f|^{2}-\alpha f_{t}\right)$ then $\frac{1}{t} G=\left(\frac{|\nabla u|^{2}}{u^{2}}-\alpha \frac{u_{t}}{u}\right)$. The approach is also by using cut-off function and estimating $\left(\Delta-\partial_{t}\right)(t \varphi G)$ at the point where the maximum value for $(\varphi G)$ is attained as we did in Theorem 2.1. The argument follows;

$$
\left(\Delta-\partial_{t}\right)(t \varphi G)=2 t \nabla \varphi \nabla G-\varphi G+t \varphi\left(\Delta-\partial_{t}\right) G+t G\left(\Delta-\partial_{t}\right) \varphi
$$

Suppose $(\varphi G)$ attains its maximum value at $\left(x_{0}, t_{0}\right) \in M \times[0, T]$, for $t_{0}>0$. Since $(\varphi G)(x, 0)=0$ for all $x \in M$, we have by derivative test that

$$
\nabla(\varphi G)\left(x_{0}, t_{0}\right)=0, \quad \frac{\partial}{\partial t}(\varphi G)\left(x_{0}, t_{0}\right) \geq 0, \quad \Delta(\varphi G)\left(x_{0}, t_{0}\right) \leq 0
$$

where the function $(\varphi G)$ is being considered with support on $\mathcal{Q}_{2 \rho} \times[0, T]$ and we have assumed that $(\varphi G)\left(x_{0}, t_{0}\right)>0$ for $t_{0}>0$. By $(3.9)$ we notice that

$$
\left(\Delta-\partial_{t}\right)(\varphi G)\left(x_{0}, t_{0}\right) \leq 0 .
$$

Using (3.8)-(3.9) and the last lemma, we have

$$
\begin{aligned}
0 \geq\left(\Delta-\partial_{t}\right)(t \varphi G) & \geq 2 t \nabla \varphi \nabla G-\varphi G \\
& +t \varphi\left\{-2\langle\nabla f, \nabla G\rangle-\left(|\nabla f|^{2}-\alpha \partial_{t} f\right)-2 \alpha t k_{1}|\nabla f|^{2}\right. \\
& \left.+\frac{2 \alpha}{n p} t\left(|\nabla f|^{2}-\partial_{t} f\right)^{2}-\frac{\alpha n q}{2} t \widehat{K}\right\} \\
& +t G\left(\Delta-\partial_{t}\right) \varphi
\end{aligned}
$$


where $\widehat{K}=\left(k_{1}+k_{2}\right)^{2}$. Noticing also that $\varphi \nabla G$ can be replaced be $-G \nabla \varphi$ by the condition $\nabla(\varphi G)=0$. Therefore

$$
\begin{aligned}
0 \geq & 2 t \nabla \varphi \nabla G-\varphi G+2 t \nabla f \nabla \varphi \cdot G \\
& \left.+t \varphi\left\{\frac{2 \alpha}{n p} t\left(|\nabla f|^{2}-\partial_{t} f\right)^{2}-2 \alpha t k_{1}|\nabla f|^{2}-\frac{\alpha n q}{2} t \widehat{K}\right)\right\} \\
& -t \varphi\left(|\nabla f|^{2}-\alpha \partial_{t} f\right)+t G\left(\Delta-\partial_{t}\right) \varphi . \\
0 \geq & -2 t \frac{|\nabla \varphi|^{2}}{\varphi} G-2 \varphi G-2 t|\nabla f||\nabla \varphi| G \\
& \left.+t \varphi\left\{\frac{2 \alpha}{n p} t\left(|\nabla f|^{2}-\partial_{t} f\right)^{2}-2 \alpha t k_{1}|\nabla f|^{2}-\frac{\alpha n q}{2} t \widehat{K}\right)\right\} \\
& +t G\left(\Delta-\partial_{t}\right) \varphi .
\end{aligned}
$$

As we have noted earlier, Calabi's trick and Laplacian comparison theorem allows us to do the following calculation on the cut-off function depending on the geodesic distance, since we know that cut locus does not intersect with the geodesic cube

$-\frac{|\nabla \varphi|^{2}}{\varphi} \geq-\frac{C_{2}}{\rho^{2}}, \quad \Delta \varphi \geq-\frac{C_{1}}{\rho} \sqrt{k_{1}}-\frac{C_{2}}{\rho^{2}}$ and $-\frac{\partial \varphi}{\partial t} \geq-C_{2} \widehat{K}-\frac{C_{1}}{\tau}, \tau \in(0, T]$.

Hence

$$
t G\left(\Delta-\partial_{t}\right) \varphi \geq t C_{3}\left(-\frac{1}{\rho} \sqrt{k_{1}}-\frac{1}{\rho^{2}}-\frac{1}{\tau}-\widehat{K}\right),
$$

where we have taken $C_{3}$ to be the maximum of $C_{1}, C_{2}$, so our computation becomes

$$
\begin{aligned}
0 \geq & -2 t \frac{C_{2}}{\rho^{2}} G-2 \varphi G-2 t \frac{\sqrt{C_{2}}}{\rho}|\nabla f| \varphi^{\frac{1}{2}} G \\
& +t \varphi\left\{\frac{2 \alpha}{n p} t\left(|\nabla f|^{2}-\partial_{t} f\right)^{2}-2 \alpha t k_{1}|\nabla f|^{2}-\frac{\alpha n q}{2} t \widehat{K}\right\} \\
& +C_{3} t\left(-\frac{1}{\rho} \sqrt{k_{1}}-\frac{1}{\rho^{2}}-\frac{1}{\tau}-\widehat{K}\right) G .
\end{aligned}
$$

Multiplying through by $0 \leq \varphi \leq 1$ again we have

$$
\left.\begin{array}{rl}
0 \geq-2 \varphi^{2} G & -2 t \frac{\sqrt{C_{2}}}{\rho}|\nabla f| \varphi^{\frac{3}{2}} G \\
+ & \frac{2 t^{2}}{n}\left\{\frac{\alpha}{p}\left(\varphi|\nabla f|^{2}-\varphi \partial_{t} f\right)^{2}-\alpha n k_{1} \varphi^{2}|\nabla f|^{2}-\frac{\alpha n^{2} q}{4} \widehat{K} \varphi^{2}\right\} \\
+ & t C_{3}\left(-\frac{1}{\rho} \sqrt{k_{1}}-\frac{1}{\rho^{2}}-\frac{1}{\tau}-\widehat{K}\right)(\varphi G)
\end{array}\right\} .
$$


Using a standard argument from Li and Yau [17], see also Schoen and Yau [20], we let

$$
y=\varphi|\nabla f|^{2} \text { and } Z=\varphi f_{t} \text { to have } \varphi^{2}|\nabla f|^{2}=\varphi y \leq y
$$

and

$$
y^{\frac{1}{2}}(y-\alpha z)=\varphi^{\frac{1}{2}}|\nabla f|\left(\varphi|\nabla f|^{2}-\varphi \partial_{t} f\right)=\frac{1}{t}|\nabla f| \varphi^{\frac{3}{2}} G .
$$

Noticing that

$$
\left.\begin{array}{rl}
(y-z)^{2} & =\left[\frac{1}{\alpha}(y-\alpha z)+\frac{\alpha-1}{\alpha} y\right]^{2} \\
& =\frac{1}{\alpha^{2}}(y-\alpha z)^{2}+\frac{(\alpha-1)^{2}}{\alpha^{2}} y^{2}+\frac{2(\alpha-1)}{\alpha^{2}} y(y-\alpha z)
\end{array}\right\}
$$

and

$$
\left.\begin{array}{rl}
(y-z)^{2}-n p k_{1} y- & n p \frac{\sqrt{C_{2}}}{\rho} y^{\frac{1}{2}}(y-\alpha z) \\
=\frac{1}{\alpha^{2}} & (y-\alpha z)^{2}+\left(\frac{(\alpha-1)^{2}}{\alpha^{2}} y^{2}-n p k_{1} y\right) \\
+\left(\frac{2(\alpha-1)}{\alpha^{2}} y-n p \frac{\sqrt{C_{2}}}{\rho} y^{\frac{1}{2}}\right)(y-\alpha z)
\end{array}\right\}
$$

using the inequality of the form $a x^{2}-b x \geq-\frac{b^{2}}{4 a},(a, b>0)$, we have

$$
\frac{(\alpha-1)^{2}}{\alpha^{2}} y^{2}-n p k_{1} y \geq-\frac{\alpha^{2} n^{2} p^{2} k_{1}^{2}}{4(\alpha-1)^{2}}, \quad \frac{2(\alpha-1)}{\alpha^{2}} y-n p \frac{\sqrt{C_{2}}}{\rho} y^{\frac{1}{2}} \geq-C_{2} \frac{\alpha^{2} n^{2} p^{2}}{8(\alpha-1) \rho^{2}} .
$$

Putting together (3.11) - (3.13), the second and the third terms in the right hand side of (3.10) are further computed as follows, using $t(y-\alpha z)=\varphi G$;

$$
\begin{aligned}
\frac{2 t^{2}}{n}\left\{\frac{\alpha}{p}(y-z)^{2}-\alpha n k_{1} y-\frac{\alpha n^{2} q}{4} \widehat{K} \varphi^{2}-\frac{n \sqrt{C_{2}}}{\rho} y^{\frac{1}{2}}(y-\alpha z)\right\} \\
=\frac{2 t^{2}}{n}\left\{\frac{\alpha}{p}\left((y-z)^{2}-n p k_{1} y-\frac{n p \sqrt{C_{2}}}{\rho} y^{\frac{1}{2}}(y-\alpha z)\right)-\frac{\alpha n^{2} q}{4} \widehat{K} \varphi^{2}\right\} \\
=\frac{2 t^{2}}{n}\left\{\frac { \alpha } { p } \left[\frac{1}{\alpha^{2}}(y-\alpha z)^{2}+\left(\frac{(\alpha-1)^{2}}{\alpha^{2}} y^{2}-n p k_{1} y\right)\right.\right. \\
\left.\left.+\left(\frac{2(\alpha-1)}{\alpha^{2}} y-n p \frac{\sqrt{C_{2}}}{\rho} y^{\frac{1}{2}}\right)(y-\alpha z)\right]-\frac{\alpha n^{2} q}{4} \widehat{K} \varphi^{2}\right\}
\end{aligned}
$$




$$
\geq \frac{2 t^{2}}{n}\left\{\left(\frac{1}{\alpha p}(y-\alpha z)^{2}-\frac{\alpha^{3} n^{2} p k_{1}^{2}}{4(\alpha-1)^{2}}\right)-\left(\frac{C_{2} \alpha^{3} n^{2} p}{8 \rho^{2}(\alpha-1)}(y-\alpha z)+\frac{\alpha n^{2} q}{4} \widehat{K} \varphi^{2}\right)\right\}
$$

Hence by (3.10)

$$
\begin{aligned}
0 \geq-2 \varphi^{2} G & +\left\{\frac{2 t^{2}}{n}\left(\frac{1}{\alpha p}(y-\alpha z)^{2}-\frac{\alpha^{3} n^{2} p k_{1}^{2}}{4(\alpha-1)^{2}}\right)-\frac{2 t}{n}\left(\frac{C_{2} \alpha^{3} n^{2} p}{8 \rho^{2}(\alpha-1)}(\varphi G)\right)\right. \\
& \left.-\frac{\alpha n q}{2} t^{2} \widehat{K} \varphi^{2}\right\}+C_{3} t\left(-\frac{1}{\rho} \sqrt{k_{1}}-\frac{1}{\rho^{2}}-\frac{1}{\tau}-\widehat{K}\right)(\varphi G)
\end{aligned}
$$

since $t(y-\alpha z)=\varphi G$, we have

$$
\begin{aligned}
0 \geq \frac{2}{\alpha n p}(\varphi G)^{2} & +\left[\frac{C_{4}}{\rho^{2}} t\left(-\frac{p \alpha^{3}}{(\alpha-1)}-\rho \sqrt{k_{1}}-1-\frac{\rho^{2}}{\tau}-\rho^{2} \widehat{K}\right)-1\right](\varphi G) \\
& -\left(\frac{\alpha^{3} n p k_{1}^{2}}{2(\alpha-1)^{2}} t^{2}+\frac{\alpha n q}{2} t^{2} \widehat{K} \varphi^{2}\right)
\end{aligned}
$$

where $C_{4}$ depends on $n$. We see that the left hand side of the last inequality is a quadratic polynomial in $(\varphi G)$, then using the quadratic formula and elementary inequality of the form $\sqrt{1+a^{2}+b^{2}} \leq 1+a+b$ yields

$$
\begin{gathered}
\varphi G \leq \frac{\alpha n p}{4}+\frac{\alpha n p}{4 \rho^{2}} C_{4} t\left(\frac{p \alpha^{3}}{(\alpha-1)}+\rho \sqrt{k_{1}}+\frac{\rho^{2}}{\tau}+\rho^{2} \widehat{K}\right) \\
+\frac{\alpha n p}{4}\left(\frac{2 \alpha k_{1}}{\alpha-1} t+2 \widehat{K}^{\frac{1}{2}} \varphi t \sqrt{\frac{q}{p}}\right) \\
=\frac{\alpha n p}{4}+\frac{\alpha n p}{4 \rho^{2}} C_{4} t\left(\frac{p \alpha^{3}}{(\alpha-1)}+\frac{\rho^{2}}{\tau}+\rho^{2} \widehat{K}\right)+\frac{\alpha^{2} n p k_{1}}{2(\alpha-1)} t+\frac{\alpha n}{2} t \widehat{K}^{\frac{1}{2}} \sqrt{p q} .
\end{gathered}
$$

Recall that we picked up $\varphi(x, t)$ such that $0 \leq \varphi \leq 1$ and particularly $\varphi(x, t)=1$ in $\mathcal{Q}_{2 \rho}$ and since $\left(x_{0}, t_{0}\right)$ is a maximum point for $(\varphi G)$ in $\mathcal{Q}_{2 \rho}$, we have $G(x, \tau)=$ $(\varphi G)(x, \tau) \leq(\varphi G)\left(x_{0}, t_{0}\right)$. Hence

$$
\frac{1}{t} G(x, t) \leq \frac{\alpha n p}{4 t}+\frac{\alpha n}{4 \rho^{2}} C_{4}\left(\frac{\alpha p}{(\alpha-1)}+\frac{\rho^{2}}{\tau}+\rho^{2} \widehat{K}\right)+\frac{\alpha^{2} n p}{2(\alpha-1)} k_{1}+\frac{\alpha n}{2} \widehat{K}^{\frac{1}{2}} \sqrt{p q}
$$

for all $x \in M$ such that $\operatorname{dist}\left(x, x_{0}, \tau\right)<\rho$ and since $\tau \in(0, T]$ was arbitrarily chosen. This formula implies the estimate (3.7) which was to be proved. 
Let $M$ be an $n$-dimensional compact (or noncompact without boundary) manifold with bounded Ricci curvature, using previous Lemma 3.1 and local gradient Theorem 3.2, we now present global estimates for the positive solutions to the heat equation when the metric evolves by the Ricci flow.

\section{Theorem 3.3. (Global Estimates)}

Let $(M, g(t))$ be a complete manifold and $g(t)$ solves the Ricci flow (3.1) such that its Ricci curvature is bounded for all $(x, t) \in M \times[0, T]$. Suppose $u=$ $u(x, t)>0$ is any positive solution to the heat equation (3.2), then we have for $-k_{1} g(x, t) \leq R c(x, t) \leq k_{2} g(x, t)$ and $\alpha>1$ with $\frac{1}{p}+\frac{1}{q}=\frac{1}{\alpha}$

$$
\frac{|\nabla u|^{2}}{u^{2}}-\alpha \frac{u_{t}}{u} \leq \frac{\alpha n p}{4 t}+\frac{\alpha^{2} n p}{2(\alpha-1)} k_{1}+\frac{\alpha n}{2}\left(k_{1}+k_{2}\right) \sqrt{p q}
$$

for all $(x, t) \in M \times[0, T]$.

Moreover, if we assume $M$ has nonnegative Ricci curvature in the case of compact manifold, i.e., for $0 \leq R c(x, t) \leq k g(x, t)$, we have

$$
\frac{|\nabla u|^{2}}{u^{2}}-\alpha \frac{u_{t}}{u} \leq \frac{\alpha n p}{4 t}+\frac{\alpha n}{2} k \sqrt{p q}
$$

for all $(x, t) \in M \times[0, T]$ and $\alpha \geq 1$ with $\frac{1}{p}+\frac{1}{q}=\frac{1}{\alpha}$.

The extreme case when $\alpha=1$ is the cheapest to prove where we choose $p=q=2 \alpha$ and the result immediately yields

$$
\frac{|\nabla u|^{2}}{u^{2}}-\frac{u_{t}}{u} \leq \frac{n}{2 t}+n k
$$

Proof. Set $f=\log u$ and allow $G$ to remain as before, calculating at the maximum point $\left(x_{0}, t_{0}\right) \in M \times(0, T]$, we will show that a new function

$$
\widetilde{G}=G-\frac{\alpha^{2} n p}{2(\alpha-1)} t k_{1}-\frac{\alpha n}{2} t\left(k_{1}+k_{2}\right) \sqrt{p q}
$$

satisfies the inequality

$$
\widetilde{G} \leq \frac{\alpha n p}{4}+\frac{\alpha^{2} n p}{2(\alpha-1)} t k_{1}+\frac{\alpha n}{2} t\left(k_{1}+k_{2}\right) \sqrt{p q} .
$$

It suffices to prove that $\widetilde{G} \leq \alpha n p$ for any $(x, t) \in M \times(0, T]$ and the inequality (4.4) will follow at once. We now show this by contradiction. Suppose $\widetilde{G}>\alpha n p$ and that $\widetilde{G}$ has its maximum at the point $\left(x_{0}, t_{0}\right)$, then we know that

$$
\nabla \widetilde{G}\left(x_{0}, t_{0}\right)=0, \quad \Delta \widetilde{G}\left(x_{0}, t_{0}\right) \leq 0, \quad \frac{\partial}{\partial t} \widetilde{G}\left(x_{0}, t_{0}\right) \geq 0, \text { and }\left(\Delta-\partial_{t}\right) \widetilde{G}\left(x_{0}, t_{0}\right) \leq 0
$$


then by Lemma 3.1, we have $0 \geq\left(\Delta-\partial_{t}\right) \widetilde{G} \geq\left(\Delta-\partial_{t}\right) G$. Noticing that

$$
\begin{aligned}
\left(|\nabla f|^{2}-f_{t}\right)^{2} & =\frac{1}{\alpha^{2}}\left(|\nabla f|^{2}-\alpha f_{t}\right)^{2}+\frac{2(\alpha-1)}{\alpha^{2}}\left(|\nabla f|^{2}-\alpha f_{t}\right)|\nabla f|^{2}+\frac{(\alpha-1)^{2}}{\alpha}|\nabla f|^{4} \\
& =\frac{1}{\alpha^{2}}\left(\frac{G}{t_{0}}\right)^{2}+\frac{2(\alpha-1)}{\alpha^{2}}|\nabla f|^{2}\left(\frac{G}{t_{0}}\right)+\frac{(\alpha-1)^{2}}{\alpha}|\nabla f|^{4} .
\end{aligned}
$$

By Lemma 3.1

$$
\begin{aligned}
0 \geq\left(\Delta-\partial_{t}\right) G \geq & -2 \nabla f, \nabla G+\frac{2 \alpha}{n p} t_{0}\left(|\nabla f|^{2}-\partial_{t} f\right)^{2}-\left(|\nabla f|^{2}-\alpha \partial_{t} f\right) \\
& -2 \alpha t_{0} k_{1}|\nabla f|^{2}-\frac{\alpha n q}{2} t_{0}\left(k_{1}+k_{2}\right)^{2} .
\end{aligned}
$$

Following the calculation in Theorem 3.2 we obtain

$$
\frac{G}{t_{0}} \leq \frac{\alpha n p}{4 t_{0}}+\frac{\alpha^{2} n p}{2(\alpha-1)} k_{1}+\frac{\alpha n}{2}\left(k_{1}+k_{2}\right)^{2} \sqrt{p q}
$$

after sending $\rho$ to $\infty$. Consequently, we have the following inequality

$$
0 \geq \frac{2 t_{0}}{\alpha n p}\left(\frac{G}{t_{0}}\right)^{2}-\frac{G}{t_{0}}-\left(\frac{\alpha^{2} n p}{2(\alpha-1)} k_{1}+\frac{\alpha n}{2}\left(k_{1}+k_{2}\right)^{2} \sqrt{p q}\right)
$$

resulting into a quadratic inequality, and since from (3.18)

$$
\begin{gathered}
\frac{G}{t_{0}}=\frac{\widetilde{G}}{t_{0}}+\frac{\alpha^{2} n p}{2(\alpha-1)} k_{1}+\frac{\alpha n}{2}\left(k_{1}+k_{2}\right)^{2} \sqrt{p q}, \\
\frac{2 t_{0}}{\alpha n p}\left(\frac{G}{t_{0}}\right)^{2}-\frac{G}{t_{0}}-\frac{\alpha^{2} n p}{2(\alpha-1)} k_{1} \leq 0 .
\end{gathered}
$$

Using the quadratic formula, we have

$$
\frac{G}{t_{0}} \leq \frac{\alpha n p}{4 t_{0}}\left\{1+\sqrt{1+\frac{4 \alpha n p}{(\alpha-1)} k_{1}}\right\},
$$

which obviously implies that $\widetilde{G} \leq \alpha n p$, a contradiction by the assumption (3.19). By definition of $\widetilde{G}$, we therefore have

$$
\frac{G}{t_{0}}=\frac{|\nabla u|^{2}}{u^{2}}-\frac{u_{t}}{u} \leq \frac{\widetilde{G}}{t_{0}}+\frac{\alpha^{2} n p}{2(\alpha-1)} k_{1}+\frac{\alpha n}{2}\left(k_{1}+k_{2}\right) \sqrt{p q} .
$$

The desired estimate follows since $t_{0}$ was arbitrarily chosen. 
Corollary 3.4. Let $(M, g(t))$ be a complete manifold and $g(t)$ solves the Ricci flow (3.1) such that its Ricci curvature satisfies $-k_{1} g(x, t) \leq R c(x, t) \leq$ $k_{2} g(x, t)$ for all $(x, t) \in M \times[0, T]$. Let $u=u(x, t)>0$ be any positive solution to the heat equation (3.2), then, for $\alpha>1$ with $\frac{1}{p}+\frac{1}{q}=\frac{1}{\alpha}$ we have

$$
\frac{|\nabla u|^{2}}{u^{2}}-\alpha \frac{u_{t}}{u} \leq \frac{\alpha n p}{4 t}+C(n) \alpha^{2}\left(k_{1}+k_{2}\right)
$$

for all $(x, t) \in M \times[0, T]$ and absolute constant $C(n)$ depending on $n$ only.

\section{Harnack Inequalities and Estimates}

As an application of our gradient estimate in the last section, we obtain some Harnack estimates for all positive solutions to the heat equation under the Ricci flow. We choose to follow the traditional approach of integrating Harnack quantity along a geodesic path connecting two points and exponentiating the result. Given $x_{1}, x_{2} \in M$ and $t_{1}, t_{2} \in[0, T]$ satisfying $t_{1}<t_{2}$

$$
\Theta\left(x_{1}, t_{1} ; x_{2}, t_{2}\right)=\inf _{\gamma} \int_{t_{1}}^{t_{2}}\left(\left|\frac{d}{d t} \gamma(t)\right|^{2}+\frac{4}{A} h\right) d t
$$

where the infimum is taken over all the smooth path $\gamma:\left[t_{1}, t_{2}\right] \rightarrow M$ connecting $x_{1}$ and $x_{2}$. The norm $|$.$| depends on t$. We now present a lemma whose aim is to give an insight into how the approach goes. (See [2] and [20]).

Lemma 4.1. Let $(M, g(t))$ be a complete solution to the Ricci flow. Let $u: M \times[0, T] \rightarrow \mathbb{R}$ be a smooth positive solution to the heat equation (1.1) and $h$ be a $C^{2}$-function on $M \times[0, T]$. Define $f=-\log u$ and assumed that

$$
-\frac{\partial f}{\partial t} \leq \frac{1}{A}\left(\frac{B}{t}+C-|\nabla f|^{2}\right)+h, \quad(x, t) \in M \times[0, T]
$$

for some $A, B, C>0$. Then, the inequality

$$
u\left(x_{1}, t_{1}\right) \leq u\left(x_{2}, t_{2}\right)\left(\frac{t_{2}}{t_{1}}\right)^{\frac{B}{A}} \exp \left(\frac{A}{4} \Theta\left(x_{1}, t_{1} ; x_{2}, t_{2}\right)+\frac{C}{A}\left(t_{2}-t_{1}\right)\right)
$$

holds for all $\left(x_{1}, t_{1}\right)$ and $\left(x_{2}, t_{2}\right)$ such that $t_{1}<t_{2}$.

Proof. Obtain the time differential of a function $f$ depending on the path $\gamma$ as follows $\left(t \in\left[t_{1}, t_{2}\right]\right)$,

$$
\frac{d}{d t} f(\gamma(t), t)=\nabla f(\gamma(t), t) \frac{d}{d t} \gamma(t)-\left.\frac{\partial}{\partial s} f(\gamma(t), s)\right|_{s=t}
$$




$$
\begin{aligned}
& \leq|\nabla f|\left|\frac{d}{d t} \gamma(t)\right|+\frac{1}{A}\left(\frac{B}{t}+C-|\nabla f|^{2}\right)+h \\
& \leq \frac{A}{4}\left|\frac{d}{d t} \gamma(t)\right|^{2}+\frac{B}{A t}+\frac{C}{A}+h .
\end{aligned}
$$

The last inequality was obtained by the application of completing the square method in form of a quadratic inequality satisfying $a x^{2}-b x \geq-\frac{b^{2}}{4 a}, \quad(a, b>0)$. Then integrating over the path from $t_{1}$ to $t_{2}$, we have

$$
\begin{aligned}
f\left(x_{2}, t_{2}\right)-f\left(x_{1}, t_{1}\right) & =\int_{t_{1}}^{t_{2}} \frac{d}{d t} f(\gamma(t), t) d t \\
& \leq \frac{A}{4} \int_{t_{1}}^{t_{2}}\left|\frac{d}{d t} \gamma(t)\right|^{2} d t+\left.\frac{B}{A} \log t\right|_{t_{1}} ^{t^{2}}+\frac{C}{A}\left(t_{2}-t_{1}\right)+\int_{t_{1}}^{t_{2}} h d t .
\end{aligned}
$$

The required estimate (4.1) follows immediately after exponentiation.

Theorem 4.2. Let $(M, g(t))$ be a complete manifold and $g(t)$ solves the Ricci flow (3.1) such that $-k_{1} g(x, t) \leq R c(x, t) \leq k_{2} g(x, t)$ for some constants $k_{1}, k_{2}>0$ and all $(x, t) \in M \times[0, T]$. Suppose $u(x, t)$ is any positive solution to the heat equation (3.2), then for any points $\left(x_{1}, t_{1}\right)$ and $\left(x_{2}, t_{2}\right)$ in $M \times[0, T]$ such that $0<t_{1}<t_{2} \leq T$, we have for any $\alpha>1$ with $\frac{1}{p}+\frac{1}{q}=\frac{1}{\alpha}$

$$
\frac{u\left(x_{1}, t_{1}\right)}{u\left(x_{2}, t_{2}\right)} \leq\left(\frac{t_{2}}{t_{1}}\right)^{\frac{n p}{4}} \exp \left\{\frac{\alpha \Theta\left(x_{1}, t_{1} ; x_{2}, t_{2}\right)}{4\left(t_{2}-t_{1}\right)}+\frac{\left(t_{2}-t_{1}\right)}{2(\alpha-1)} A\left(n, \alpha, p, q, k_{1}, k_{2}\right)\right\}
$$

where

$$
\Theta\left(x_{1}, t_{1} ; x_{2}, t_{2}\right)=\inf \int_{0}^{1}|\dot{\gamma}(s)|^{2} d s
$$

the infimum is taken over all the smooth paths $\gamma:\left[t_{1}, t_{2}\right] \rightarrow M$ connecting $x_{1}$ and $x_{2}$, and $A\left(n, \alpha, p, q, k_{1}, k_{2}\right)=n \alpha p k_{1}+n(\alpha-1)\left(k_{1}+k_{2}\right) \sqrt{p q}$.

Theorem 4.3. Let $(M, g(t))$ be a compact Riemannian manifold evolving by the Ricci flow. Let $u(x, t)$ be a positive solution to the heat equation for $t \in(0, T]$, if the Ricci curvature is nonnegatively bounded by $0 \leq R c \leq K g$, then for $0<t_{1}<t_{2} \leq T$, we have for any $\alpha>1$ with $\frac{1}{p}+\frac{1}{q}=\frac{1}{\alpha}$

$$
\frac{u\left(x_{1}, t_{1}\right)}{u\left(x_{2}, t_{2}\right)} \leq\left(\frac{t_{2}}{t_{1}}\right)^{\frac{n p}{4}} \exp \int_{0}^{1}\left(\frac{\alpha|\dot{\gamma}(s)|^{2}}{4\left(t_{2}-t_{1}\right)}+C(n) \frac{\left(t_{2}-t_{1}\right)}{2(\alpha-1)} K \sqrt{p q}\right) d s
$$

where $C(n)$ is some constant depending only on $n$. 


\section{Proof. (of Theorem 4.2)}

Let $\gamma:[0,1]$ be a minimizing geodesic connecting points $x_{1}$ and $x_{2}$ in $M$ such that $\gamma(0)=x_{2}$ and $\gamma(1)=x_{1}$ with $\left|\gamma^{\prime}(s)\right|$ being the length of the vector $\gamma^{\prime}(s)$ at time $\tau(s)=\left((1-s) t_{2}+s t_{1}\right), \quad 0 \leq t_{1}<t_{2} \leq T$. Define $\eta(s)=$ $\log u\left(\gamma(s),(1-s) t_{2}+s t_{1}\right)$, clearly, $\eta(1)=\log u\left(x_{1}, t_{1}\right)$ and $\eta(0)=\ln u\left(x_{2}, t_{2}\right)$.

Integrating $\left(\frac{\partial}{\partial s} \log u\right)$ along $\eta(s)$, we obtain

$$
\log u\left(x_{1}, t_{1}\right)-\log u\left(x_{2}, t_{2}\right)=\int_{0}^{1}\left(\frac{\partial}{\partial s} \log u(s)\right) d s
$$

i.e.,

$$
\log \left(\frac{u\left(x_{1}, t_{1}\right)}{u\left(x_{2}, t_{2}\right)}\right)=\left.\log (\gamma(t), t)\right|_{0} ^{1} .
$$

By direct computation, we have on the path $\gamma(s)$ that

$$
\begin{aligned}
\frac{\partial}{\partial s} \eta(s)=\frac{d}{d s} \log u & =\nabla \log u \cdot \dot{\gamma}(s)-\frac{\partial}{\partial t} \log u \\
& =\frac{\nabla u}{u} \cdot \dot{\gamma}(s)-\frac{u_{t}\left(t_{2}-t_{1}\right)}{u} \\
& =\left(t_{2}-t_{1}\right)\left(\frac{\nabla u}{u} \cdot \frac{\dot{\gamma}(s)}{t_{2}-t_{1}}-\frac{u_{t}}{u}\right) .
\end{aligned}
$$

From Theorem 3.3, we have

$$
-\frac{u_{t}}{u} \leq-\frac{|\nabla u|^{2}}{\alpha u^{2}}+\frac{\alpha n p}{2(\alpha-1)} k_{1}+\frac{n}{2}\left(k_{1}+k_{2}\right) \sqrt{p q}+\frac{n p}{4 t} .
$$

Therefore

$$
\begin{aligned}
\frac{d}{d s} \log u \leq & \frac{\left(t_{2}-t_{1}\right)}{\alpha}\left\{\alpha \frac{\nabla u}{u} \cdot \frac{\dot{\gamma}(s)}{t_{2}-t_{1}}-\frac{|\nabla u|^{2}}{u^{2}}\right\} \\
& \quad+\frac{\left(t_{2}-t_{1}\right)}{2(\alpha-1)}\left(n \alpha p k_{1}+n(\alpha-1)\left(k_{1}+k_{2}\right) \sqrt{p q}\right)+\left(t_{2}-t_{1}\right) \frac{n p}{4 t} \\
= & \frac{\left(t_{2}-t_{1}\right)}{\alpha}\left(\frac{\nabla u}{u}-\frac{\dot{\gamma}(s)}{\left(t_{2}-t_{1}\right)}\right)^{2}+\frac{\left(t_{2}-t_{1}\right)}{\alpha} \cdot \frac{\alpha^{2}}{4\left(t_{2}-t_{1}\right)^{2}}|\dot{\gamma}|^{2} \\
& \quad+\frac{\left(t_{2}-t_{1}\right)}{2(\alpha-1)}\left(n \alpha p k_{1}+n(\alpha-1)\left(k_{1}+k_{2}\right) \sqrt{p q}\right)+\left(t_{2}-t_{1}\right) \frac{n p}{4 t} \\
\leq & \frac{\alpha|\dot{\gamma}|^{2}}{4\left(t_{2}-t_{1}\right)}+\frac{\left(t_{2}-t_{1}\right)}{2(\alpha-1)}\left(n \alpha p k_{1}+n(\alpha-1)\left(k_{1}+k_{2}\right) \sqrt{p q}\right)+\left(t_{2}-t_{1}\right) \frac{n p}{4 t} .
\end{aligned}
$$


Integrating the last inequality over the path $\gamma(s)$, we get

$$
\begin{array}{r}
\log \frac{u\left(x_{1}, t_{1}\right)}{u\left(x_{2}, t_{2}\right)}=\int_{0}^{1} \log u(\gamma(s), \tau(s)) d s \leq \int_{0}^{1}\left\{\frac{\alpha|\dot{\gamma}(s)|^{2}}{4\left(t_{2}-t_{1}\right)}\right. \\
\left.+\frac{\left(t_{2}-t_{1}\right)}{2(\alpha-1)}\left(n \alpha p k_{1}+n(\alpha-1)\left(k_{1}+k_{2}\right) \sqrt{p q}\right)\right\} d s \\
+\left(t_{2}-t_{1}\right) \frac{n p}{4} \int_{0}^{1} \frac{d s}{(1-s) t_{2}+s t_{1}},
\end{array}
$$

which at once yield the required Harnack estimate by simply exponentiating.

Proof. (of Theorem 4.3) We can follow Theorem 3.3 on condition $0 \leq$ $R c \leq K g$ to obtain the Harnack inequality

$$
\frac{|\nabla u|^{2}}{u^{2}}-\alpha \frac{u_{t}}{u} \leq \frac{\alpha n p}{4 t}+\frac{\alpha n}{2} k \sqrt{p q} \quad \text { with } \quad \alpha \geq 1 \text { and } \frac{1}{p}+\frac{1}{q}=\frac{1}{\alpha} .
$$

with $\alpha \geq 1$ and $\frac{1}{p}+\frac{1}{q}=\frac{1}{\alpha}$. Calculating as in the proof of Theorem 4.2, the desired Harnack estimate follows immediately.

Corollary 4.4. With the same hypothesis and notation as in Theorem 4.3 , it holds for any $\alpha>1$ that

$$
\frac{u\left(x_{1}, t_{1}\right)}{u\left(x_{2}, t_{2}\right)} \leq\left(\frac{t_{2}}{t_{1}}\right)^{\frac{\alpha n}{2}} \exp \int_{0}^{1}\left(\frac{\alpha|\dot{\gamma}(s)|^{2}}{4\left(t_{2}-t_{1}\right)}+C(n) \frac{\alpha\left(t_{2}-t_{1}\right)}{2(\alpha-1)} K\right) d s
$$

where $C(n)$ is some constant depending only on $n$.

\section{Acknowledgements}

The author acknowledges the anonymous referees whose comments led to an improvement over the first draft of this article. His research is supported by FGN/TETFUND. 


\section{References}

[1] D. Bakry and Z. M. Qian, Harnack inequalities on a manifold with positive or negative Ricci curvature, Revista Matematica Iberoamericana, 15, No. 1 (1999), 143-179, doi: 10.4171/RMI/253.

[2] M. Băileşteanu, X. Cao, and A. Pulemotov, Gradient estimates for the heat equation under the Ricci flow, J. of Funct. Analysis, 258, No. 10 (2010), 3517-3542, doi: 10.1016/j.jfa.2009.12.003.

[3] E. Calabi, An extension of E. Hopfs maximum principle with an application to Riemannian geometry, Duke Math. J., 25, No. 1 (1958), 45-56, doi: 10.1215/S0012-7094-58-02505-5.

[4] X. Cao, Differential Harnack estimates for backward heat equations with potentials under the Ricci flow, J. Funct. Anal., 255, No. 4 (2008), 10241038, doi: 10.1016/j.jfa.2008.05.009.

[5] X. Cao and R. S. Hamilton, Differential Harnack estimates for timedependent heat equations with potentials, Geom. Funct. Anal., 19, No. 4 (2009), 989-1000, doi: 10.1007/s00039-009-0024-4.

[6] J. Cheeger and S-T. Yau, A lower bound for the heat kernel, Comm. Pure Appl. Math., 34, No. 4 (1981), 465-480, doi: 10.1002/cpa.3160340404.

[7] B. Chow, The Ricci flow on 2-sphere, J. Differential Geom., 33, No. 2 (1991), 325-334, doi: euclid.jdg/1214446319.

[8] B. Chow and D. Knopf, The Ricci flow: An introduction. AMS, Providence, RI (2004).

[9] B. Chow, P. Lu and L. Ni, Hamilton's Ricci Flow: An Introduction. American Mathematics Society, (2006).

[10] K. Ecker, D. Knopf, L. Ni and P. Topping, Local monotonicity and mean value formulas for evolving Riemanniann manifolds, J. reine angew. Math., 616, (2008), 89-130, doi: 10.1515/CRELLE.2008.019.

[11] C. Guenther, The fundamental solution on manifolds with timedependent metrics, J. Geom. Anal., 12, No. 3 (2002), 425-436, doi: 10.1007/BF02922048.

[12] R. Hamilton, The Ricci flow on Surfaces, Contemp. Math., AMS, Providence, RI 71, (1988), 237-262, doi: 10.1090/conm/071/954419. 
[13] R. Hamilton, A matrix Harnack estimate for the heat equation, Comm. Anal. Geom., 1, No. 1 (1993), 113-126.

[14] R. Hamilton, Monotonicity formulas for parabolic flows on manifolds, Commun. Anal. Geom., 1, No. 1 (1993), 127-137.

[15] R. Hamilton, The formation of singularities in the Ricci flow, In Surveys in differential geometry, 2 (Cambridge, MA, 1993), 7136. Internat. Press, Cambridge, MA, (1995), doi: 10.4310/SDG.1993.v2.n1.a2.

[16] S. Kuang and Qi S. Zhang, A gradient estimate for all positive solutions of the conjugate heat equation under Ricci flow, J. Funct. Anal., 55, No. 4 ( 2008), 1008-1023, doi: 10.1016/j.jfa.2008.05.014.

[17] P. Li and S-T. Yau, On the parabolic kernel of the Schrödinger operator, Acta Math., 156, No. 3-4 (1986), 153-201, doi: 10.1007/BF02399203.

[18] S. Liu, Gradient estimate for solutions of the heat equation under Ricci flow, Pacific J. Math., 243, No. 1 (2009), 165-180, doi: 10.2140/pjm.2009.243.165.

[19] G. Perelman, The entropy formula for the Ricci flow and its geometric application, ArXiv: math.DG/0211159v1 (2002).

[20] R. Schoen and S.-T. Yau, Lectures on differential geometry, International Press, Cambridge, MA (1994).

[21] Qi. S. Zhang, Some gradient estimates for the heat equation on domains and for an equation by Perelman, Int. Math. Res. Not, 2006, Article ID 92314, (2006), 1-39, doi: 10.1155/IMRN/2006/92314. 
